\title{
Hypoglycemia, CTCAE
}

National Cancer Institute

\section{Source}

National Cancer Institute. Hypoglycemia, CT CAE. NCI Thesaurus. Code C143567.

A disorder characterized by laboratory test results that indicate a low concentration of

glucose in the blood. 